\title{
Quiste sinovial en el tendón del músculo tibial posterior. Caso clínico, consideraciones biomecánicas y revisión bibliográfica
}

\author{
Synovial Cyst in the Posterior Tibial Muscle Tendon. \\ Case Report, Biomechanical Considerations and Literature Review
}

\author{
José Valero Salas ${ }^{1}$, José Gallart Ortega², David González LóPez ${ }^{3}$ \\ ${ }^{1}$ Podólogo y Antropólogo. Especialista en Cirugía Podológica. Doctor por la Universidad de Zaragoza (Sociología), \\ Doctor por la Universidad de Zaragoza (Medicina). \\ ${ }^{2}$ Podólogo y Antropólogo. Doctor por la Universidad de Zaragoza (Medicina). \\ ${ }^{3}$ Podólogo. Máster por la Universidad Complutense de Madrid. Doctorando por la Universidad de Zaragoza
} (Medicina).

\author{
Correspondencia: \\ José Valero Salas \\ Centro Podologico José valero Salas \\ C/ Coso 55 \\ E-50001 Zaragoza \\ Correo electrónico: clinicajosevalero@gmail.com
}

Fecha de recepción: 31 de enero de 2014

Fecha de aceptación: 30 de abril de 2014

Los autores declaran no tener ningún tipo de interés económico o comercial.

\section{RESUMEN}

Se presenta un caso de quiste sinovial localizado en la cara interna del tobillo derecho de una paciente que presenta pies pronados de articulación subastragalina con el antepié patológicamente compensado en supinación. Se describe el procedimiento quirúrgico, el tratamiento postquirúrgico y el estudio anatomopatológico. Al mismo se hacen algunas consideraciones acerca de la etiología patomecánica de los quistes sinoviales y una revisión bibliográfica de los tumores benignos y malignos que afectan a las vainas tendinosas y a las membranas sinoviales.

Palabras clave: quiste sinovial; ganglión; cirugía; biomecánica; patomecánica; podiatría.

\section{ABSTRACT}

A case of synovial cyst located on the inner side of the right ankle of a patient presenting joint subtalar pronate foot with forefoot pathologically compensated in supination is presented. Describes the surgical procedure, postsurgical treatment and the histopathological study. At the same time which are made some considerations about the pathomechanical etiology of synovial cysts and a literature review of benign and malignant tumors that affect the tendon sheaths and synovial membranes.

Key words: synovial cyst; ganglial cyst; surgery; biomechanics; pathomechanics; podiatry.

Sumario: 1. Introducción. 2. Caso clínico. 3. Procedimiento quirúrgico. 4. Tratamiento post-quirúrgico. 5. Estudio anatomopatológico. 6. Discusión. Bibliografía.

Referencia normalizada: Valero Salas, J., Gallart Ortega, J., González López, D. Quiste sinovial en el tendón del músculo tibial posterior. Caso clínico, consideraciones biomecánicas y revisión bibliográfica. Rev. Int. Cienc. Podol. 2014; 8(2): 99-108. 


\section{INTRODUCCIÓN}

Los quistes sinoviales tipo ganglión son lesiones muy comunes ${ }^{1}$. Normalmente ocurren en la muñeca, donde se reconocen fácilmente, aunque se presentan en otras localizaciones y, con gran frecuencia, en el pie, donde se presenta más habitualmente (aunque puede hacerlo en diversas zonas del pie $)^{2,3,4}$ en la articulación del tobillo, en la articulación de Chopart y en la articulación de Lisfranc. Cuando se presenta en el tobillo, el quiste sinovial puede comprimir alguna rama el nervio tibial posterior y provocar un dolor que puede ser diagnosticado erróneamente de un síndrome del túnel tarsiano o de un síndrome compartimental o, como se han descrito casos, enmascarar una tumoración maligna ${ }^{5}$.

El denominado común e indistintamente quiste sinovial o ganglión engloba en realidad dos entidades perfectamente diferenciadas:

1. El pseudoquiste sinovial (ganglial cyst), que es una tumefacción llena de líquido viscoso, desarrollado alrededor de las vainas tendinosas así como en las epífisis óseas. Es el resultado de una degeneración mucoide del tejido conjuntivo para-articular o para-tendinoso. Se diferencia histológicamente con el quiste sinovial en que carece de una capa de células sinoviales.

2. El quiste sinovial propiamente dicho (synovial cyst) que es una expansión de la membrana sinovial de las grandes articulaciones como la cadera y la rodilla, aunque también puede afectar a las vainas tendinosas y a las bolsas serosas.

La descripción anatomopatológica típica del quiste sinovial describe una estructura de tejido fibroadiposo en cuyo seno se aprecia la pared de una cavidad quística parcialmente colagenizada y revestida por una capa de células muy aplanadas que ha desaparecido en buena parte de su extensión. Subyacentemente se aprecian algunas luces vasculares y un discreto infiltrado inflamatorio linfoplasmocitario de distribución preferentemente perivascular.

De un modo esquemático, el músculo tibial posterior se origina en la cara posterior y superior de la tibia y en la cara interna del peroné, y se inserta en el navicular, en el calcáneo, en los tres cuneiformes y en las bases de los metatarsianos segundo, tercero y cuarto. Desde un punto de vista funcional, el músculo tibial posterior tiene una acción aductora muy enérgica al traccionar del navicular medialmente y es extensor de las articulaciones tibio-peroneo-astragalina y mediotarsiana. Merced a sus expansiones plantares es un músculo supinador, desempeñando un papel fundamental en el sostén y en la orientación de la bóveda plantar. Por tanto, su función es eminentemente extensora y antipronadora.

La patología más frecuente del músculo tibial posterior son las tendinopatias producidas por alteraciones biomecánicas y más habituales por la actividad deportiva ${ }^{6}$. En este último sentido, la disfunción del tibial posterior es una de las patologías más habituales en corredores, especialmente en los corredores de fondo. En una carrera de larga duración, se ve sometido a un gran esfuerzo de forma repetida lo puede producir tendinitis, tenosinovitis y tendinosis. Se ha propuesto diversos factores que provocan tendinopatías del músculo tibial posterior. Entre éstas se encuentra la inflamación crónica de etiología reumáti$\mathrm{ca}^{7}$, traumatismos ${ }^{8}$, iatrogenias (infiltraciones y cirugías previas utilizadas para el tratamiento de esta patología $)^{9,10}$, las ya citadas alteraciones patomecánicas ${ }^{11}$ y las formas mixtas, en especial la enfermedad reumática unida a pies planos-valgos ${ }^{12}$.

Los tumores y lesiones paratumorales de la sinovial son considerados como poco frecuentes en el pie pero potencialmente destructivos y, en el caso de los histológicamente malignos, pueden pasar desapercibidos y, por tanto, ser diagnosticados de modo tardío o ser confundidos con otras patologías o tumores benignos. Presentamos un caso de quiste sinovial afectando al tendón del tibial posterior en todos sus aspectos: clínico, métodos de diagnóstico por imagen, tratamiento quirúrgico y estudio anatomopatológico. Al mismo tiempo que se hacen algunas consideraciones biomecánicas acerca de la etiología de este tumor y se repasan los aspectos más significativos de los tumores malignos que afectan a las vainas tendinosas y a las membranas sinoviales.

\section{CASO CLÍNICO}

Paciente, mujer de 69 años de edad que, desde hace más de un año, presenta un nódulo dolo- 
roso situado por debajo del maléolo tibial, en la zona calcáneo-astragalina medial del pie derecho (Figura 1). La tumoración le produce dolor al caminar y a la flexo-extensión del tobillo en reposo y una moderada impotencia funcional. La exploración física muestra una masa concreta y uniforme, del color de la piel, de consistencia dura, movible con facilidad y dolorosa a la presión y al pinzamiento lateral.

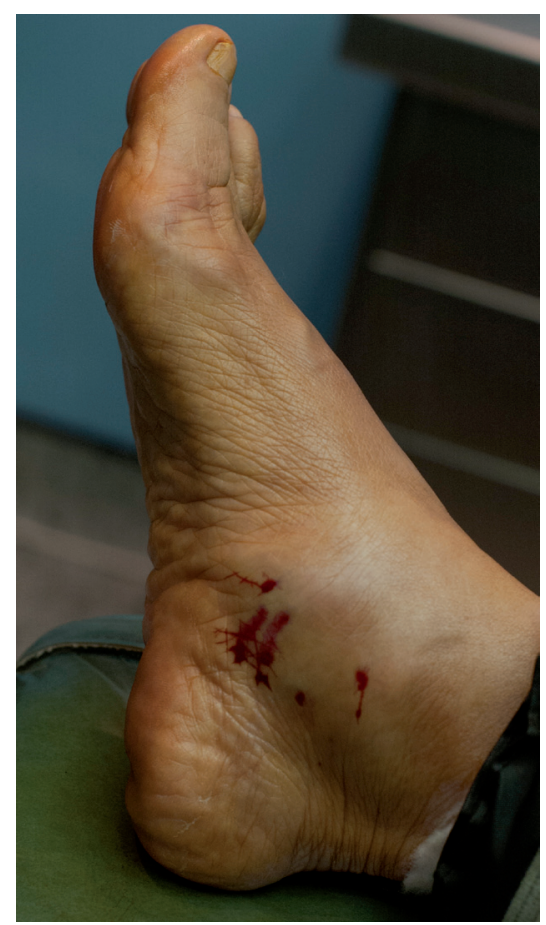

Figura 1a. Aspecto de la lesión. Vista panorámica.

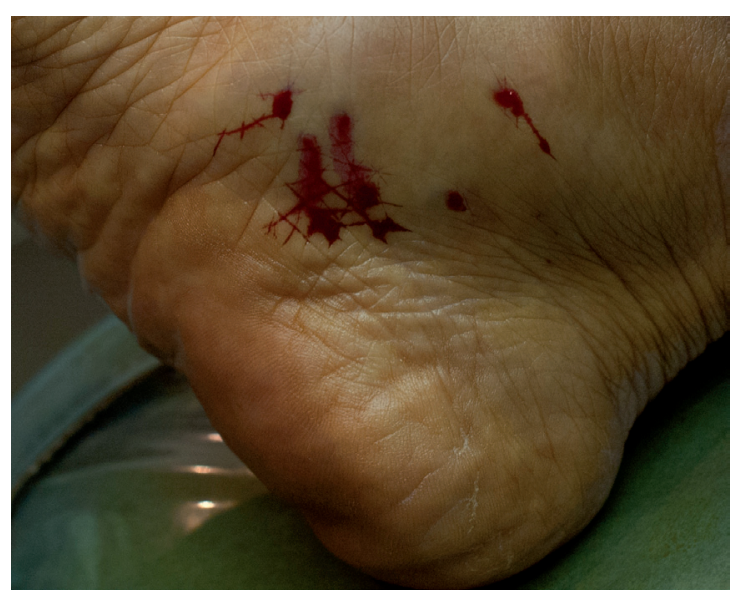

Figura 1b. Aspecto de la lesión. Detalle.
Ante la naturaleza y evolución dudosas del proceso, se solicita estudio ultrasonográfico, que muestra al tendón tibial posterior aumentado de grosor con una ligera cantidad de líquido en la vaina a nivel retromaleolar proximal e inframaleolar a nivel proximal algo denso y a nivel inframaleolar más líquido pero con formaciones tipo vellosidades en el interior de la vaina, todo ello compatible con tenosinovitis calcificada (Figura 2).

Como antecedentes significativos hay que destacar que fue intervenida, hace más de veinte años, de hallux abductus valgus (osteotomía distal tipo Austin), síndrome de sobrecarga de

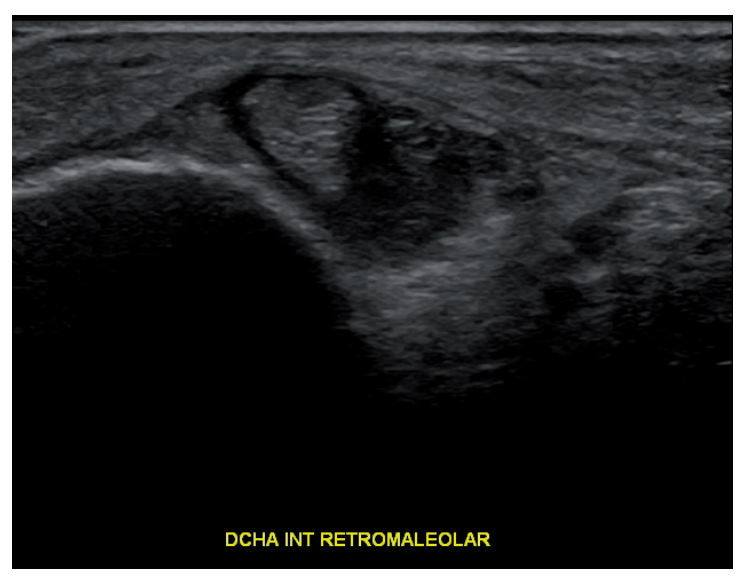

Figura 2a. Imagen ecográfica retromaleolar en la que se aprecia la colección líquida en el interior de la vaina del tendón del tibial posterior.

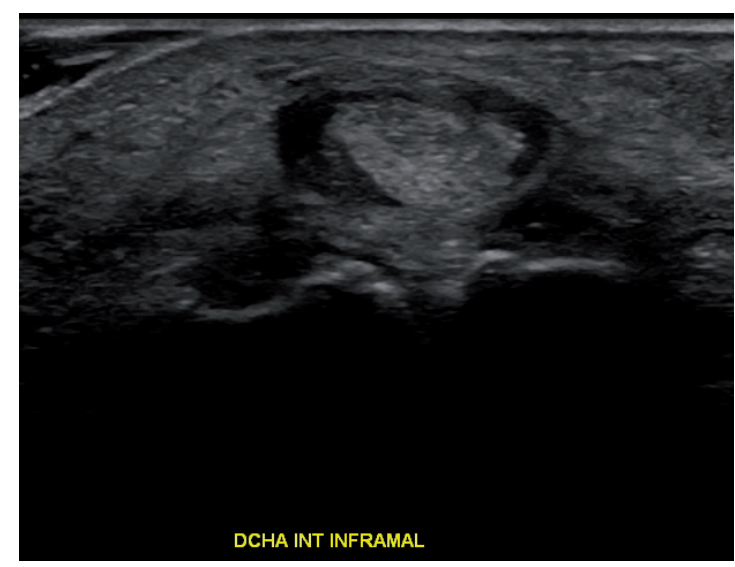

Figura 2b. Imagen ecográfica inframaleolar en la que se aprecia la colección líquida en el interior de la vaina del tendón del tibial posterior. 


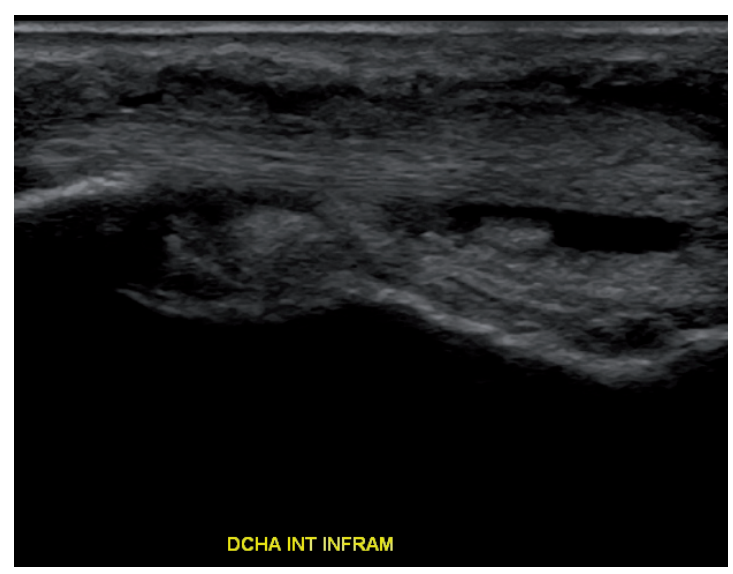

Figura 2c. Imagen ecográfica inframaleolar en la que se aprecian la vellosidades en el interior de la vaina.

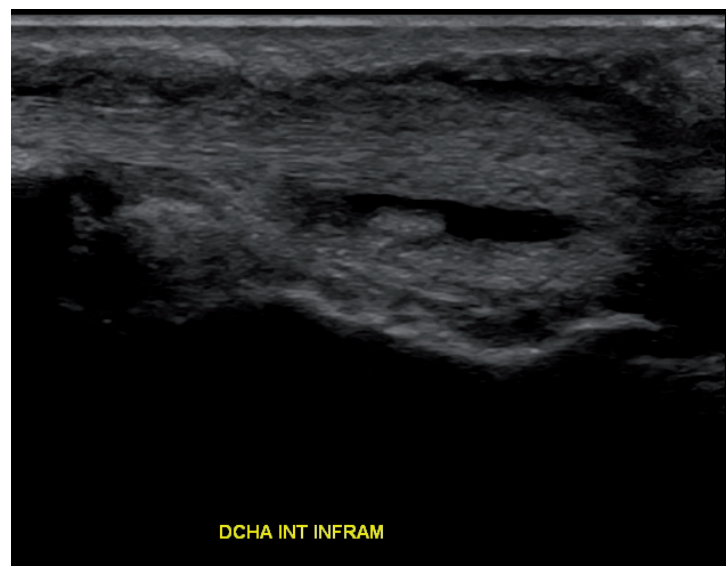

Figura 2d. Imagen ecográfica inframaleolar en la que se aprecian la vellosidades en el interior de la vaina, las calcificaciones y la hipertrofia del tendón.

segundo y tercer metatarsianos (osteotomías elevadoras), segundo y tercer dedos en marti1lo (artroplastias). A la exploración biomecánica, presenta pies pronados de articulación subastragalina con el antepié patológicamente compensado en supinación. Sus alteraciones biomecánicas están siendo compensadas con tratamiento ortopodológico.

Como persisten los dolores y la evolución de la tumoración, de acuerdo con la historia clínica, ha sido muy corta en el tiempo, se opta por el tratamiento quirúrgico.

\section{PROCEDIMIENTO QUIRÚRGICO}

Previa anestesia local de la zona a intervenir con mepivacaína al $1 \%$ y hemostasia supramaleolar, se practica una incisión longitudinal, justo por encima de la lesión, abarcándola en su totalidad y con un margen de seguridad de $1 \mathrm{~cm}$ proximal y $1 \mathrm{~cm}$ distal (Figura $3 \mathrm{a}$ ). Se procede seguidamente a profundizar la incisión teniendo especial cuidado en preservar la integridad de la tumoración durante todo el proceso de disección (Figura 3b) hasta la extracción completa de la tumoración con su cápsula que debe ser extirpada en su totalidad (Figura 3c y 3d). El cierre de la herida se realiza por planos: sutura absorbible de 3/0 en los planos internos (Figura 3e) y monofilamento plástico de $4 / 0$ en la piel (Figura 3f).

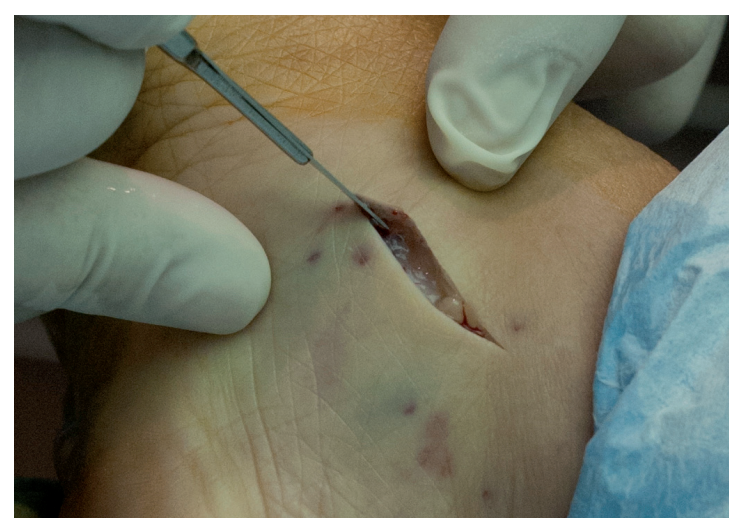

Figura 3a. Cirugía de un quiste sinovial. Incisión.

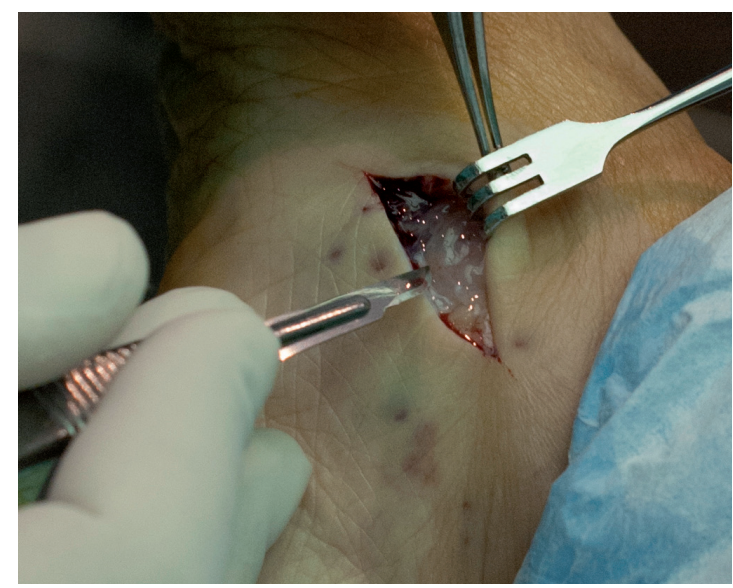

Figura 3b. Cirugía de un quiste sinovial. Disección. 


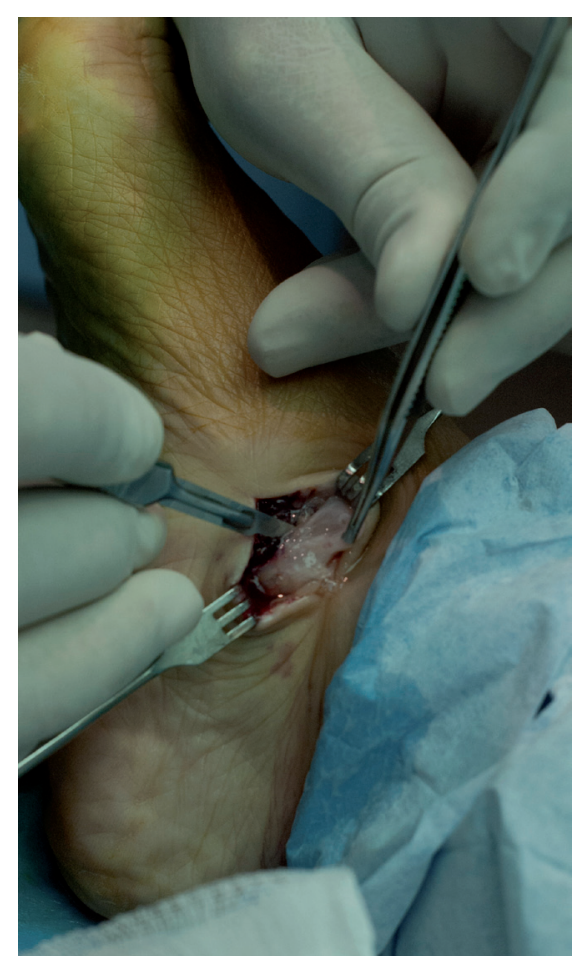

Figura 3c. Cirugía de un quiste sinovial. Disección profunda.

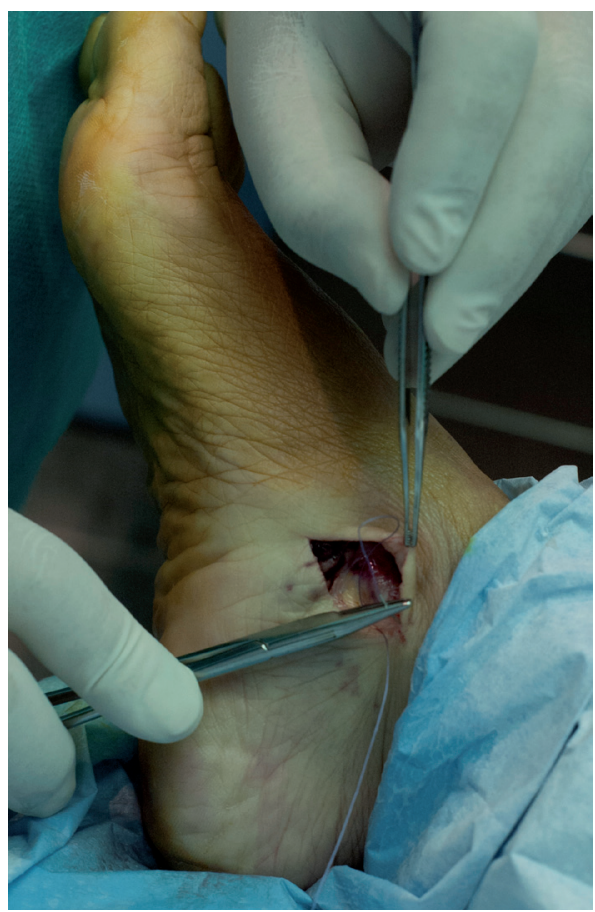

Figura 3e. Cirugía de un quiste sinovial. Sutura interna.

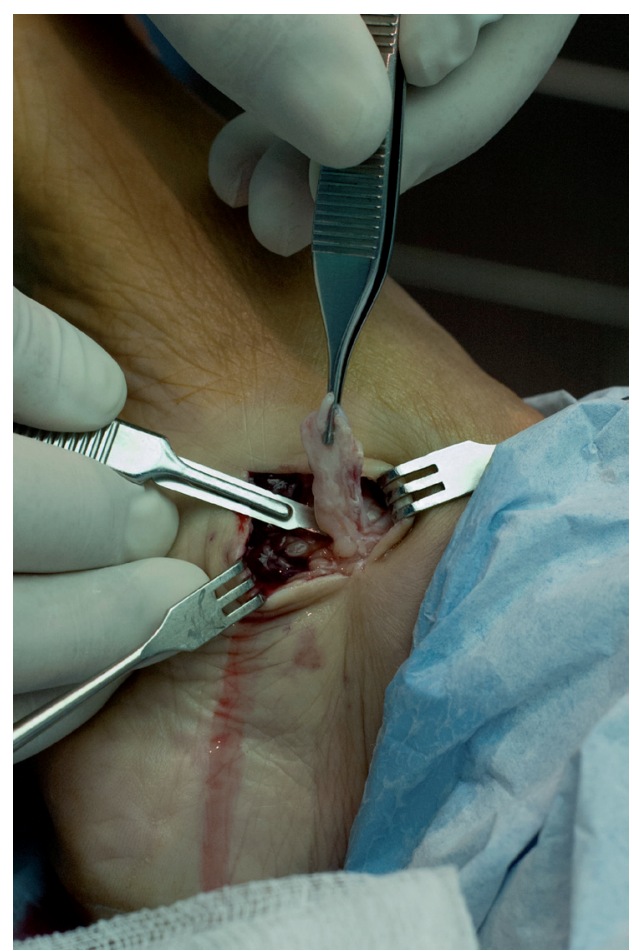

Figura 3d. Cirugía de un quiste sinovial. Extracción del quiste.

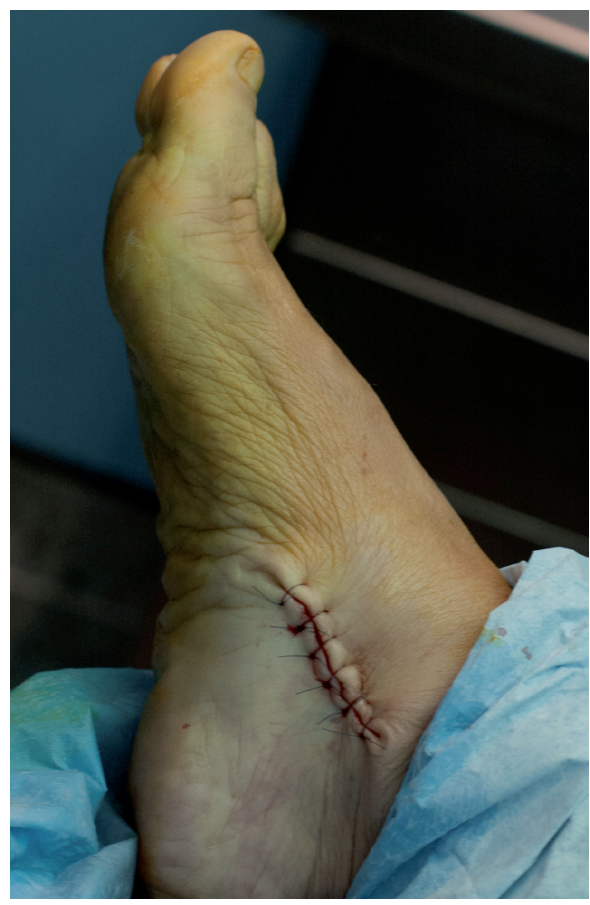

Figura 3f. Cirugía de un quiste sinovial. Sutura de la piel. 


\section{TRATAMIENTO POST-QUIRÚRGICO}

La pieza extraída se remitió para su estudio anatomopatológico.

Se prescribió antibioterapia por vía oral (500 mg de amoxicilina cada 8 horas) durante ocho días. Se indicó reposo absoluto durante las primeras veinticuatro horas y relativo durante los días siguientes hasta la retirada de la sutura no absorbible, que tuvo lugar a los 12 días de la intervención. Se mantuvo un vendaje semicompresivo en la zona, con venda adhesiva y elástica, con la finalidad de mantener estable la movilidad del tobillo evitando movimientos de hiper-pronación. Este vendaje se renovó semanalmente durante cuatro semanas más, momento en el que se revisó su tratamiento ortopodológico compensador consistente en soportes plantares de polipropileno con poste posterior y elemento de contención lateral externo y se le dio el alta quirúrgica. La paciente, en la actualidad, es revisada de su tratamiento ortopodológico cada seis meses sin haberse producido recidiva del quiste sinovial a los 18 meses de la intervención.

\section{ESTUDIO ANATOMOPATOLÓGICO}

Descripción macroscópica: Un fragmento que mide $2 \times 0,7 \times 0,5 \mathrm{~cm}$ con aspecto de tejido fibroadiposo. Se incluye en su totalidad (Figura 4).

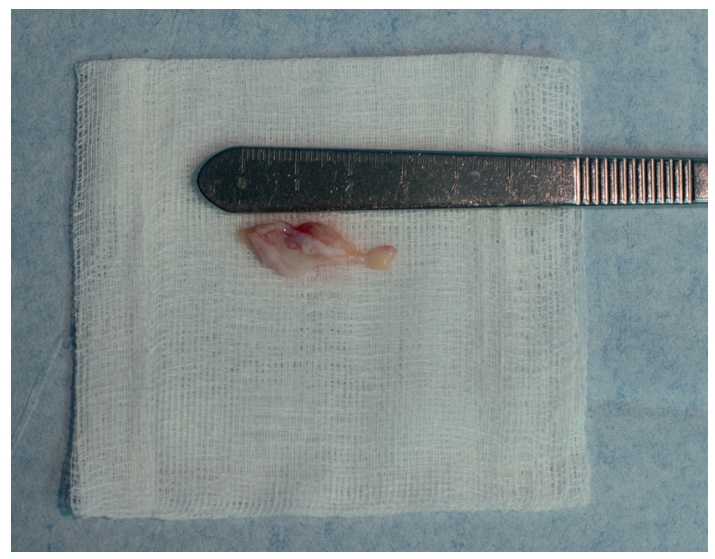

Figura 4. Cirugía de un quiste sinovial. Pieza extirpada.
Descripción microscópica: Las secciones estudiadas están constituidas por tejido fibro-adiposo en cuyo seno se observa una estructura quística revestida, en parte, por epitelio aplanado (Figura $5)$.

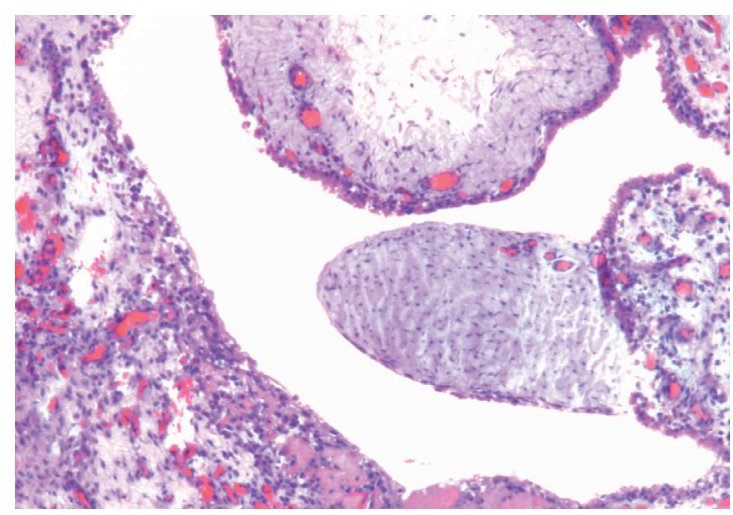

Figura 5a. Microfotografía del quiste sinovial (H$\mathrm{E}, \mathrm{x} 100)$

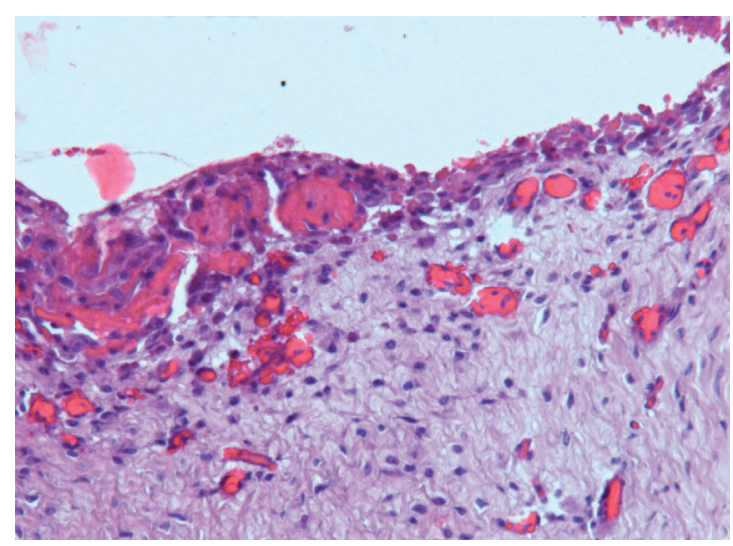

Figura 5b. Microfotografía del quiste sinovial (HE, $x 200$ )

Diagnóstico anatomopatológico: Biopsia de cara interna del tobillo derecho con un quiste de tipo sinovial.

\section{DISCUSIÓN}

En el diagnóstico por imagen de los tumores sinoviales, sean éstos benignos o malignos, la radiografía simple es poco sensible, sin embargo la resonancia magnética nuclear y la ecografía son unos métodos muy sensibles para determi- 
nar la localización, forma y extensión (nunca para establecer, de una manera categórica su tipología histopatológica) ${ }^{13}$. No obstante, hay que tomar como primera y fundamental referencia diagnóstica una meticulosa exploración y una completa anamnesis ya que el desarrollo de los tumores malignos que afectan a la piel y a las partes blandas afectan especialmente a personas con antecedentes previos de haber padecido dichos tumores ${ }^{14}$.

Los quistes sinoviales son lesiones paratumorales que, con mucha frecuencia, desaparecen espontáneamente o con procedimientos paliativos. Sin embargo, cuando los procedimientos paliativos o incruentos fracasan es preciso recurrir a la cirugía, cuyos resultados no siempre son seguros, incluso extirpándolos en su totalidad. La causa del fracaso quirúrgico, incluso con una extirpación en su totalidad, vendría dada por no reparar la causa etiológica, es decir, por no reparar la herniación de la vaina tendinosa, causante de la extravasación de mucina, origen del ganglión y la alteración funcional que la produjo. En este sentido, estudios anatomopatológicos demuestran que la disfunción del tendón del músculo tibial posterior, como el caso presentado, puede producir tendinitis ${ }^{15,16}$, tendinosis y fibrosis con o sin rotura de dicho tendón ${ }^{17}$. Con mucha frecuencia la causa patomecánica de la hernia tendinosa (y consiguientemente del quiste sinovial) está muy alejada del lugar donde se produce esta lesión paratumoral por lo que un adecuado tratamiento ortopodológico y/o rehabilitador son determinantes para evitar recidivas postquirúrgicas.

Por tanto, el quiste sinovial es un típico ejemplo de los tumores de etiología patomecánica ${ }^{18}$. La disfunción del músculo tibial posterior produce alteraciones en la correcta función del pie $\mathrm{y}$ ha sido estudiada por varios autores ${ }^{19,20}$. En el caso presentado el quiste sinovial tiene una etiología claramente causada por la disfunción del tendón del tibial posterior asociada/ provocada a/por un pie pronado en la articulación subastragalina $\mathrm{y}$, además, prolongada en el tiempo (por el engrosamiento del tendón, como determinó el estudio ecográfico). Si existe una gran cantidad de métodos de tratamiento conservador de la disfunción de la disfunción del tendón del tibial posterior $^{8,21,17,22}$ y de la articulación subastragalina pronada y patológicamente compensada en supinación de antepié, la estructuración de la alteración funcional de la paciente del caso presentado (de 69 años), con una articulación astrágalo-navicular prácticamente rígida, impiden aplicar en su totalidad los objetivos terapéuticos propios de la disfunción del tendón del tibial posterior que son, siguiendo a Pascual et al., controlar el dolor, favorecer la remodelación tisular, evitar la aparición o progresión de la deformidad, mejorar la alineación antepié/ retropié y la funcionalidad del paciente ${ }^{23}$. En el caso presentado, se ha podido constatar que la utilización de soportes plantares (estabilizadores, compensadores y/ o correctores) para el tratamiento del pie pronado y para la disfunción del músculo tibial posterior (que ha sido propuesta y utilizada con éxito por numerosos autores $^{8,24,25,26}$ ) sirvió para el doble objetivo de compensar las alteraciones en la biomecánica de los pies y evitar la recidiva del quiste sinovial.

Los términos ganglión y quiste sinovial se utilizan como sinónimos con mucha frecuencia. Ello es debido a la similitud histopatológica de ambos procesos ${ }^{27}$. No obstante se tendrá muy en cuenta que en el nombre genérico de ganglión se pueden solapar otras tumoraciones y entidades paratumorales: ciertos tumores de vainas tendinosas como el hemangioma sinovial, el quiste digital mucoso, los tumores de células gigantes, bursitis calcificadas ${ }^{28}$, etc. Por otra parte, desde un punto de vista epidemiológico, se tomará en consideración el hecho de que los tumores benignos y las lesiones pseudotumorales son más frecuentes que los tumores malignos de partes blandas $^{29,30}$. Sin embargo, no hay que olvidar que existen tumoraciones malignas originarias de tejido sinovial (entre las que se encuentran los sarcomas sinoviales y los condrosarcomas sinoviales) que, aunque son más frecuentes en articulaciones grandes como la rodilla ${ }^{31,32}$ y el tobillo ${ }^{33}$, se han publicado casos de sarcomas sinoviales en el pie ${ }^{34,35,36}$, en el mediopié (sarcoma sinovial monofásico) ${ }^{37}$, en los metatarsianos (en algún caso falsamente diagnosticado de neuroma de Morton $)^{38}$, en el primer dedo ${ }^{39}$, en la primera articulación metatarso-falángica (simulando una artritis gotosa $)^{40}$ y en el músculo tibial posterior ${ }^{41,42}$. Todo ello hace imprescindible un apropiado tratamiento quirúrgico ${ }^{43}$ y la remisión para estudio anatomopatológico de la tumoración extirpada. También hay que tener 
en cuenta que condromatosis sinoviales primarias pueden, en ocasiones, enmascarar un condrosarcoma sinovial ${ }^{44}$ y que se han reportado casos en los que este tumor es secundario a una condromatosis sinovial previa ${ }^{44,45,46,47}$. También se han comunicado casos en los que el sarcoma sinovial puede estar enmascarado por una infección o por un traumatismo ${ }^{36}$ y que puede ser secundario a una metástasis en otra parte del organismo, como la garganta ${ }^{48}$.

En cualquier caso, aunque está generalmente aceptado que los tumores malignos de las partes blandas del pie son raros (suponen el 1\% de todos los tumores y lesiones paratumorales que se presentan en el pie), en 2011 Nishimura et al. ${ }^{49}$ presentaron un estudio de 592 sarcomas, de los que el $28,6 \%$ correspondían a sarcomas sinoviales en el pie en el periodo 1973-2009. Este hecho, unido a la similitud clínica de los quistes sinoviales benignos y los tumores malignos que afectan a las vainas sinoviales (que no pueden ser diagnosticados con certeza por medio de los métodos diagnósticos por imagen $)^{50,51,52}$ exigen un cuidadoso diagnóstico clínico, un apropiado tratamiento quirúrgico y el estudio histopatológico del material extirpado.

\section{AGRADECIMIENTOS}

Al Dr. M.A. Marigil Gómez, MD, Anatomopatólogo, por su valiosa colaboración en el estudio anatomopatológico del caso presentado.

A la Dra. Reyes Yñigo, MD, Radióloga, por su valiosa colaboración en el estudio ecográfico del caso presentado.

\section{BIBLIOGRAFÍA}

1. Slavitt JA, Beheshti F, Lenet M, Sherman M. Ganglions of the foot. A six-year retrospective study and a review of the literature. J Am Podiatry Assoc. 1980; 70(9):459-65.

2. Barret C, Weaber TD, Schaffer SG. Ganglion Cyst of the Hallux: An Aberrant Presentation. Foot Ankle Surg. 1995;34(1):57-60.

3. Wenig JA, McCarthy DJ. Synovial cyst of the hallux. A case report. J Am Podiatr Med Assoc. 1986; 76:7-12.

4. Valero J. Il ganglione del mesopiede. Il Pod in Med. 2002;114:33-4.

5. Bernard J, Johnston L, Brennan T. Underlying synovial sarcoma in a patient with a history of complex regional pain syndrome: a case report. J Foot Ankle Surg. 2013;52(1):80-3.

6. Martín Urrialde, J.A.: Lesiones por sobrecarga del tibial posterior. Valoración funcional diferencial. Fisioterapia. 2004; 26:319-24.

7. Dubbeldam R, Baan H, Nene AV, Drossaers-Bakker KW, van de Laar MA, Hermens HJ, Buurke JH. Foot and ankle kinematics in rheumatoid arthritis: influence of foot and ankle joint and leg tendon pathologies. Arthritis Care Res (Hoboken). 2013;65(4):503-11.

8. Domínguez G, Munuera PV, Salcini JL, Palomo IC, Salcini FJ. Insuficiencia del músculo tibial posterior. Caso clínico. Rev Esp Podol. 2001;XII(1):5-11.

9. Mianiaci A, Rorabeck $\mathrm{CH}$. Compartment syndrome as a complication of repair of a hernia of the tibialis anterior: a case report. J Bone Joint Surg. 1986;68(9):1444-5.

10. Anand P, Nunley JA, DeOrio JK. Single-incision medial approach for double arthrodesis of hindfoot in posterior tibialis tendon dysfunction. Foot Ankle Int. 2013;34(3):338-44.

11. Neville C, Flemister AS, Houck J.Total and distributed plantar loading in subjects with stage II tibialis posterior tendon dysfunction during terminal stance. Foot Ankle Int. 2013;34(1):131-9.

12. Barn R, Turner DE, Rafferty D, Sturrock RD, Woodburn J. Tibialis posterior tenosynovitis and associated pes plano valgus in rheumatoid arthritis: electromyography, multisegment foot kinematics, and ultrasound features. Arthritis Care Res (Hoboken). 2013;65(4):495-502.

13. Raez R, Belezyk R, Williams RP, Fernandez MP, Zgonis T. Synovial sarcoma of the foot: a case report. J Am Podiatr Med Assoc. 2010;100(3):216-9.

14. Dominguez-Cruz JJ, Nieto-Garcia A, Rios JJ, Moreno-Ramirez D. Second non-melanoma skin cancer in Spain: frequency and Chronology. Br J Dermatol. 2013 Oct 11. doi:10.1111/bjd. 12675.

15. Blake RL, Anderson K, Ferguson H. Posterior tibial tendinitis. A literature review with case reports. J Am Podiatr Med Assoc. 1994;84(3):141-9. 
16. Rabbito M, Pohl MB, Humble N, Ferber R. Biomechanical and clinical factors related to stage I posterior tibial tendon dysfunction. J Orthop Sports Phys Ther. 2011;41(10):776-84.

17. Parsons S, Naim S, Richards PJ, McBride D. Correction and prevention of deformity in type II tibialis posterior dysfunction. Clin Orthop Relat Res. 2010;468(4):1025-32.

18. Valero J, Gallart J, González D, Marquina R, Agustín L. Tumores de origen patomecánico. Podología Clínica 2012;13(1):11-27.

19. Gluck, Heckman D, Parekh S. Tendon disorders of the foot and ankle, Part 3: The posterior tibial tendon. Am J Sports Med. 2010;38:2133.

20. Pascual R, López P, Monzó FE, García J, Herrera JM, Serrano FJ. Patomecánica de la disfunción del tendón del tibial posterior. Podología Clínica 2013;14(3):78-82.

21. Chao W, Wapner KL, Lee TH, Adams J, Hecht PJ. Nonoperative management of posterior tibial tendon dysfunction. Foot Ankle Int. 1996;17(12):736-41.

22. Bowring $\mathrm{B}$, Chockalingam $\mathrm{N}$. Conservative treatment of tibialis posterioris tendon dysfunction- A review. The Foot 2010;20:18:18-26.

23. Pascual R, López P, García J, Sánchez ME, Rodríguez C. Algorritmo de la disfunción del tendón del tibial posterior: Evidencia científica del tratamiento conservador. Podología Clínica 2013;14(4):112-20.

24. Dorca A, Céspedes T, Concustell J, Sacristán S, Dorca R. Nuestro concepto actual del pie valgo. Rev Esp Podol. 1996;VII(6):329-44.

25. Dorca A, Céspedes T, Cuevas R, Sacristán S. El deporte y la podología. Tendencias actuales en ortopodología (Primera parte). Rev Esp Podol. 1993;IV(1):6-12.

26. Dorca A, Céspedes T, Cuevas R, Sacristán S. El deporte y la podología. Tendencias actuales en ortopodología (Segunda parte). Rev Esp Podol. 1993;IV(2):83-9.

27. Alonso J, Rueda M, Rueda A. El quiste sinovial en el pie. Rev Esp Podol. 1991;II(2): 63-6.

28. Rubin LM. Calcification of a bursa beneath a heloma durum. J Am Podiatry Assoc. 1967;57(7):335.

29. Moreau G, Bush Ch, Scarborough M, Enneking W. Surgical considerations in a diagnostic imaging evaluation of musculoskeletal masses. MRI Clinics of NA. 1995;3:577-90.

30. Kransdorf M, Murphey M. Origin and classification of soft tissue tumors. In: Kransdorf M, Murphey M (Eds), Imaging of Soft Tissue Tumors. 2nd.Ed. Philadelphia: Lippincott Williams \&Wilkins;2006, pp. $1-5$.

31. Taconis W, Van der Heul R, Tamimiau A. Synovial chondrosarcoma: report of a case and review of the literature. Skeletal Radiology 1997;26:682-85.

32. Hermann G, Klein M, Abdelwahab IF, Kenan S. Synovial chondrosarcoma arising in synovial chondromatosis of the right hip. Skeletal Radiology 1997;26:366-69.

33. Hallam P, Ashwood N, Cobb J, Fazal A, Heatley W. Malignant transformation in synovial chondromatosis of knee?. Knee 2001;8: 239-42.

34. Racz R, Belczyk R, Williams RP, Fernandez MP, Zgonis T. Synovial sarcoma of the foot: a case report.J Am Podiatr Med Assoc. 2010;100(3):216-9.

35. Latt LD, Turcotte RE, Isler MH, Wong C. Case series. Soft-tissue sarcoma of the foot. Can J Surg. 2010;53(6):424-31.

36. Boc SF, Das-Wattley S, Roberts E. Synovial sarcoma arising in the foot: case report. J Foot Ankle Surg. 2011;50(1):117-21.

37. Beus J, Kreitner KF, Rompe JD, Riehle HM. Synovial sarcoma of the foot. Aktuelle Radiol. 1996;6(5):261-3.

38. Bekarev M, Elsinger EC, Villanueva-Siles E, Borzykowski RM, Geller DS. Synovial sarcoma of the foot. J Foot Ankle Surg. 2013;52(4):513-7.

39. Sutro CJ. Synovial sarcoma of the soft parts in the 1st toe: recurrence after a 35 year interval. Bull Hosp Joint Dis. 1976;37(2):105-9.

40. Du Y, Pullman-Mooar S, Schumacher HR. Synovial sarcoma of the foot mimicking acute gouty arthritis. J Rheumatol. 2005;32(10):2006-8.

41. Wertheimer SJ. Synovial sarcoma of the tibialis posterior tendon. A case report. J Am Podiatry Assoc. 1980;70(7):346-8.

42. Chou LB, Malawer MM. Synovial sarcoma presenting as posterior tibial tendon dysfunction: a report of two cases and review of the literature. Foot Ankle Int. 2004;25(11):810-4.

43. Latt LD, Turcotte RE, Islere MH, Wong C. Case series. Soft-tissue sarcoma of the foot. Can J Surg. 2010;53(6):424-31.

44. Wittkop B, Davies A, Mangham D. Primary synovial chondromatosis and synovial chondrosarcoma: 
a pictorial review. Eur Radiol. 2002; 12: 2112-9.

45. Kenan S, Abdelwahab IF, Klein M, Lewis M. Case report 817: Synovial chondrosarcoma secondary to synovial chondromatosis. Skeletal Radiology 1993; 22:623-6.

46. Ontell F, Greenspan A. Chondrosarcoma complicating synovial chondromatosis: finding with magnetic resonance imaging. Can Assoc Radiol J. 1994;45:318-23.

47. Wuisman P, Noorda R, Jutte P. Chondrosarcoma secondary to synovial chondromatosis. Report of two cases and a review of the literature. Arch Orthop Surg. 1997;116:307-11.

48. Aljubran A, Tulba A, Akhtar S. Metastatic synovial sarcoma of the foot to the throat. Am J Clin Oncol. 2008;31(2):205-6.

49. Nishimura A, Matsumine A, Asanuma K, Matsubara T, Nakamura T, Uchida A, Kato K, Sudo A. The adverse effect of an unplanned surgical excision of foot soft tissue sarcoma. World J Surg Oncol. 2011;9:160.

50. Verdugo MA. Ultrasonido en el estudio de tumores de partes blandas. Rev Chil Radiol. 2009;15(1):518.

51. Parsons T, Frink S, Campbell S. Musculoskeletal neoplasia: Helping the orthopedic surgeon establish the diagnosis. Semin Musculoskelet Radiol. 2007;11:3-15.

52. O'Connor M. Musculoskeletal imaging: what information is important for the orthopedic oncologists? Semin Musculoskelet Radiol. 2007;11:273-8. 